\title{
Robust spin transfer torque in antiferromagnetic tunnel junctions
}

\author{
Hamed Ben Mohamed Saidaoui \\ King Abdullah University of Science and Technology (KAUST), Physical Science and Engineering Division (PSE), \\ Thuwal 23955-6900, Saudi Arabia \\ Xavier Waintal \\ University Grenoble Alpes, INAC-PHELIQS, F-38000 Grenoble, France \\ CEA, INAC-PHELIQS, F-38000 Grenoble, France \\ Aurélien Manchon* \\ King Abdullah University of Science and Technology (KAUST), Physical Science and Engineering Division (PSE), \\ Thuwal 23955-6900, Saudi Arabia \\ (Received 6 July 2016; revised manuscript received 8 March 2017; published 17 April 2017)
}

\begin{abstract}
We theoretically study the current-induced spin torque in antiferromagnetic tunnel junctions, composed of two semi-infinite antiferromagnetic layers separated by a tunnel barrier, in both clean and disordered regimes. We find that the torque enabling electrical manipulation of the Néel antiferromagnetic order parameter is out of plane, $\sim \mathbf{n} \times \mathbf{p}$, while the torque competing with the antiferromagnetic exchange is in plane, $\sim \mathbf{n} \times(\mathbf{p} \times \mathbf{n})$. Here, $\mathbf{p}$ and $\mathbf{n}$ are the Néel order parameter direction of the reference and free layers, respectively. Their bias dependence shows behavior similar to that in ferromagnetic tunnel junctions, the in-plane torque being mostly linear in bias, while the out-of-plane torque is quadratic. Most importantly, we find that the spin transfer torque in antiferromagnetic tunnel junctions is much more robust against disorder than that in antiferromagnetic metallic spin valves due to the tunneling nature of spin transport.
\end{abstract}

DOI: 10.1103/PhysRevB.95.134424

\section{INTRODUCTION}

Intensive research has been achieved in the field of spin transfer torque [1-3] in ferromagnetic materials in the past two decades. Spin torque consists of the transfer of the spin angular momentum from a spin-polarized flow of conduction electrons to the local magnetic moments of a ferromagnet. This spin transfer promotes magnetic excitations resulting in magnetization switching [4-6] or self-sustained precessional motion $[7,8]$. The typical device on which spin torque switching is commonly achieved is composed of two ferromagnets separated by a spacer that can be either metallic or insulating. The former is henceforth referred to as a metallic spin valve, while the latter is called a (ferro)magnetic tunnel junction (F-MTJ). In both devices, the spin torque is dominated by an antidamping component of the form $\tau_{\|} \sim \mathbf{m} \times(\mathbf{p} \times \mathbf{m})$, where $\mathbf{p}$ is the magnetization direction of the reference layer while $\mathbf{m}$ is the magnetization direction of the free layer. In both cases, the torque is an interfacial process arising from the destructive interference between incoming electron spins with different incidences $[9,10]$. In the case of F-MTJs, a fieldlike torque of the form $\tau_{\perp} \sim \mathbf{m} \times \mathbf{p}$ also emerges, which can be as large as $10 \%$ to $30 \%$ of the in-plane torque [10-14], as confirmed experimentally [15-17]. The bias dependence of these two torque components can be tuned by engineering the junction structural asymmetry $[13,18,19]$ or in the presence of interfacial electron-magnon scattering [20].

A few years ago, the presence of spin transfer torque in metallic antiferromagnetic spin valves was predicted theoretically [21]. The authors considered a structure composed of two antiferromagnetic layers spaced by a normal metal in analogy

\footnotetext{
*aurelien.manchon@kaust.edu.sa
}

with the ferromagnetic spin valve. Experimentally, the search for current-induced torque in antiferromagnetic layers has been carried out by analyzing the alteration that occurs at the level of the exchange bias between the ferromagnetic and the antiferromagnetic layers in a conventional ferromagnetic spin valve [22,23]. However, not much progress has been realized experimentally since then due to the significant difficulty of maintaining sizable torques in these structures, as well as controlling and detecting independently the Néel order parameter dynamics. As a matter of fact, it was recently shown $[24,25]$ that even a small amount of disorder dramatically reduces the magnitude of the torque. Indeed, in order to preserve large current-driven torques in antiferromagnetic spin valves, the staggered spin density built up in the reference antiferromagnetic layer has to be transported coherently to the free antiferromagnetic layer. Disorder breaks translational invariance and prevents the coherent transmission of this staggered spin density through the spin valve.

A solution to this issue is to generate local torques, i.e., spin currents and densities that do not need to be transmitted from one part of the device to another. Several strategies have been proposed to date, such as the use of antiferromagnetic domain walls [26,27] and the exploitation of spin-orbit torques [28]. Another approach is to exploit spin-dependent tunneling transport (see Ref. [29]), which is much less sensitive to momentum scattering. Recently, tunneling anisotropic magnetoresistance has been reported in IrMn/MgO junctions [30,31], demonstrating the high quality that can be achieved in such systems. Antiferromagnetic spintronics presents tremendous potential for applications and is now gaining significant momentum [32].

In the present work, we investigate spin transfer torques in antiferromagnetic tunnel junctions (AF-MTJs). We study the voltage dependence of the spin torque components for a 
(a)

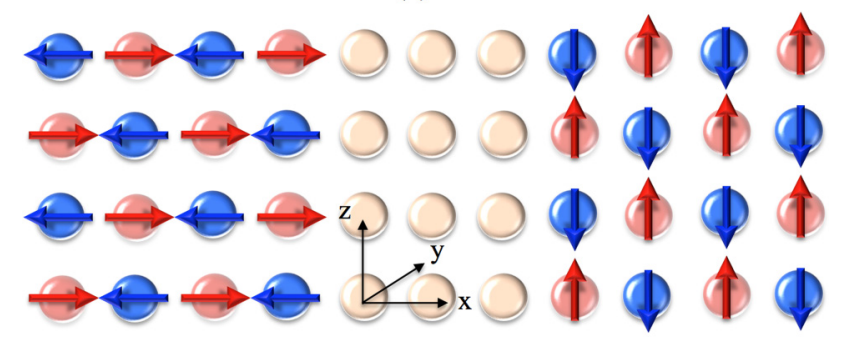

(b)

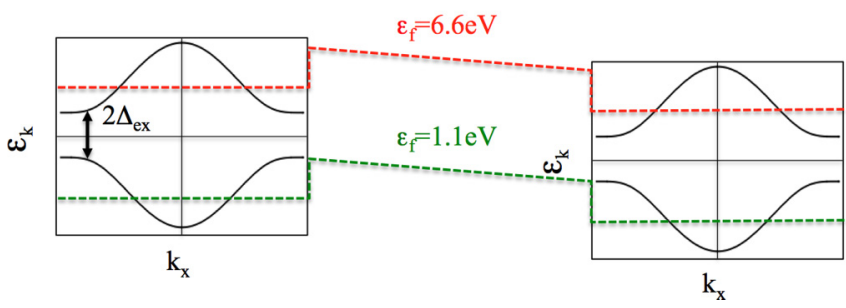

FIG. 1. (a) Schematic of the AF-MTJ consisting of two semiinfinite antiferromagnets spaced by a tunnel barrier. Red and blue atoms refer to the two antiferromagnetically coupled sublattices. (b) Illustration of the energy spacial profile and its alteration by the applied voltage. Dashed red and green lines represent the potential profile for two specific Fermi energies, $\epsilon_{f}=6.6 \mathrm{eV}$ and $\epsilon_{f}=1.1 \mathrm{eV}$, as explained in the text.

junction composed of symmetric antiferromagnetic electrodes. We finally explore the effect of disorder on the torque, demonstrating that the torque in AF-MTJs is much more robust against imperfections than that in antiferromagnetic metallic spin valves [24].

\section{METHODOLOGY}

\section{A. Tight-binding model}

The system we consider consists in two semi-infinite antiferromagnetic electrodes spaced by an insulating barrier (see Fig. 1). The two-dimensional antiferromagnets are square lattices in a G-type magnetic configuration, i.e., each magnetic moment is surrounded by nearest-neighbor moments of opposite direction. This configuration is different from the ones reported in previous theoretical works [21,29]), in which the authors consider L-type antiferromagnets composed of uncompensated layers with magnetic moments pointing in opposite directions. The width of the junction's layers is 20 atomic sites, while the barrier extends over three monolayers. In order to compute the transport properties of this system, we exploit the nonequilibrium Green's function formalism implemented in the tight-binding code KWANT [34], a procedure described in detail in Ref. [24]. The tight-binding Hamiltonian reads

$$
\hat{H}=\sum_{i} \epsilon_{i} \hat{c}_{i}^{+} \hat{c}_{i}-\sum_{i, i^{\prime}} t_{i, i^{\prime}} \hat{c}_{i}^{+} \hat{c}_{i^{\prime}}+\sum_{i} \Delta_{\mathrm{ex}}^{i} \hat{c}_{i}^{+} \mathbf{m}_{i} \cdot \hat{\boldsymbol{\sigma}} \hat{c}_{i} .
$$

The indices $i=\left(x_{i}, z_{i}\right)$ refer to the two-dimensional coordinates of the sites. $\epsilon_{i}$ is the on-site energy, $t_{i, i^{\prime}}=t$ is the hopping parameter between site $i$ and site $i^{\prime}$, restricted to nearest neighbors, and $\Delta_{\mathrm{ex}}^{i}$ is the exchange energy between the staggered local magnetic moment $\mathbf{m}_{i}$ at site $i$ and the itinerant electron spin $\left(\Delta_{\mathrm{ex}}^{i}=\Delta_{\mathrm{ex}}\right.$ in the antiferromagnetic electrodes and $\Delta_{\mathrm{ex}}^{i}=0$ in the barrier). $\hat{\sigma}$ is the vector of Pauli spin matrices, where the caret denotes a $2 \times 2$ matrix in spin space and $\hat{c}_{i}^{+}$is the creation operator of an electron at site $i$, such that $\hat{c}_{i}^{+}=\left(c_{i \uparrow}^{+}, c_{i \downarrow}^{+}\right)$, where $\uparrow$ and $\downarrow$ refer to the spin projection along the quantization axis. The on-site energy of the antiferromagnetic electrodes is constant and set to $\epsilon_{i}=\epsilon_{0}$. Throughout this study, the tunnel barrier has a height of $6 t$ so that the on-site energy in the barrier at zero bias reads $\epsilon_{i}=\epsilon_{0}+6 t$. The nonequilibrium regime is promoted by applying a potential difference $e V_{b}$ in the range $[-0.9 t, 0.9 t]$ across the junction. The chemical potential of the left (right) antiferromagnetic electrode reads $e \mu^{L(R)}=$ $\epsilon_{0} \pm e V_{b} / 2$. Hence, the on-site energy in the tunnel barrier reads $\epsilon_{i}=\epsilon_{0}+6 t+e V_{b}\left[1 / 2-x_{i} /\left(N_{B}-1\right)\right]$, where $N_{B}$ is the number of insulating layers and $x_{i}=0$ denotes the position of the first layer of the tunnel barrier.

\section{B. Torques and currents}

The nonequilibrium properties are computed from the lesser Green's function $\hat{G}_{i ; i^{\prime}}^{<}(\epsilon)=\sum_{l} f\left(\epsilon, \mu_{l}\right) \sum_{n} i \psi_{n, i}^{l}\left(\psi_{n, i^{\prime}}^{l}\right)^{*}$ (see Ref. [35]), $\psi_{n, i}^{l}$ being the scattering wave functions originating from lead $l$, with a Fermi-Dirac distribution $f\left(\epsilon, \mu_{l}\right)$. In the present work, we calculate the spin torque components from the local spin density $\mathbf{S}_{i}$, which reads

$$
\mathbf{S}_{i}=\frac{1}{2 \pi} \int \operatorname{Tr}_{\sigma}\left[\hat{\boldsymbol{\sigma}} \hat{G}_{i ; i}^{<}\right] d \epsilon .
$$

The integration runs over the full energy bandwidth up to the chemical potential of the left and right electrodes. The local torque at a particular lattice site reads $\boldsymbol{\tau}_{i}=2 \Delta_{\mathrm{ex}} \mathbf{m}_{i} \times \mathbf{S}_{i}$.

In antiferromagnets composed of collinear sublattices, two types of torques can be defined at the level of the diatomic unit cell: torques arising from uniform spin densities (i.e., when the spin density is equal on the two sublattices) and torques arising from staggered spin densities (i.e., when the spin density is opposite on the two sublattices). In our previous work [24], we called these two types of torque "rotating" and "exchange" torques, respectively. In systems without translational invariance, such as spin valves, both types of torques exist in principle and possess components in and out of the $(\mathbf{n}, \mathbf{p})$ plane, where $\mathbf{p}$ and $\mathbf{n}$ are the Néel order parameters of the polarizer and analyzer, respectively. At this stage, we want to point out a mistake in the discussion in our previous work, Ref. [24]. We claimed that the rotating torque (stemming from a uniform spin density) is responsible for the switching of the Néel order parameter, while the exchange torque (stemming from a staggered spin density) competes with the antiferromagnetic exchange and is therefore inefficient. This claim is incorrect since dc external magnetic fields (and, hence, uniform spin densities) are unable to manipulate the direction of Néel order parameters and result only in induced magnetization. This confusion comes from the assumption that antiferromagnetic dynamics somewhat resembles ferromagnetic dynamics, which is clearly untrue since precession about the antiferromagnetic exchange field is the driving force in antiferromagnetic dynamics [33]. 
Therefore, only staggered spin densities (producing exchange torques) are efficient in controlling the Néel order parameter direction in the stationary transport regime [32,33].

\section{Beyond the idealized model}

To conclude this section, let us stress the limitations of our model. First, spin-orbit coupling in the bulk of the materials as well as at interfaces is neglected. As a result, the electronic bands are spin degenerate and momentum scattering due to impurities is not accompanied by spin relaxation. In addition, tunneling anisotropic magnetoresistance and associated spinorbit torques are absent [30]. Second, the magnetic texture of the antiferromagnets is collinear, i.e., the spin angular momentum is a good quantum number in each metallic lead. In the case of noncollinear antiferromagnets, spin dephasing is enhanced [36] and spin-independent disorder is sufficient to induce spin relaxation, a mechanism that is absent in our study. Finally, the band structure of the antiferromagnetic leads is kept as simple as possible and no resonant states exist at the interface between the leads and the barrier. Such interfacial states have been identified in thin $\mathrm{Fe}(100) / \mathrm{MgO}(100) / \mathrm{Fe}(100)$ magnetic tunnel junctions [37,38] and, more recently, in $\mathrm{Cr}(001) / \mathrm{MgO}(001) / \mathrm{Cr}(001)$ antiferromagnetic tunnel junctions [39], associated with the surface states of Cr. Such states are believed to be responsible for tunneling magnetic coupling between the $\mathrm{Cr}$ electrodes [40] and could have an influence on spin transport.

\section{RESULTS AND DISCUSSION}

\section{A. Premises}

Before discussing the theoretical results, it is instructive to consider the band structure of a prototypical G-type antiferromagnet. The tight-binding Hamiltonian, Eq. (1), can be rewritten in the $\{|A\rangle,|B\rangle\} \otimes\{|\uparrow\rangle,|\downarrow\rangle\}$ space, where $A$ and $B$ refer to the antiferromagnetically coupled sublattices, and $\uparrow$ and $\downarrow$ refer to up and down spin projections, respectively. One obtains

$$
\begin{aligned}
\hat{h} & =\gamma_{k} \hat{\tau}_{x} \otimes \hat{1}+\Delta_{\mathrm{ex}} \hat{\tau}_{z} \otimes \hat{\sigma}_{z}, \\
\gamma_{k} & =-2 t\left(\cos k_{x} a+\cos k_{z} a\right) .
\end{aligned}
$$

Here, we chose the Néel order parameter to lie along $\mathbf{z}$, and $\hat{\boldsymbol{\tau}}$ and $\hat{\sigma}$ are Pauli spin matrices acting on the sublattice $\{|A\rangle,|B\rangle\}$ and spin $\{|\uparrow\rangle,|\downarrow\rangle\}$ subspaces, respectively. This Hamiltonian supports the eigenstates

$$
\begin{gathered}
\epsilon_{k}^{s}=s \sqrt{\gamma_{k}^{2}+\Delta_{\mathrm{ex}}^{2}}, \\
\psi_{s}^{\sigma}=\frac{1}{\sqrt{2}}\left(\sqrt{1+s \sigma \beta_{k}}|A\rangle+s \sqrt{1-s \sigma \beta_{k}}|B\rangle\right) \otimes|\sigma\rangle,
\end{gathered}
$$

and $\beta_{k}=\frac{\Delta_{\mathrm{ex}}}{\sqrt{\gamma_{k}^{2}+\Delta_{\mathrm{ex}}^{2}}}$. Here, $s=1(s=-1)$ denotes the top (bottom) band, and $\sigma=1(\sigma=-1)$ corresponds to spin up (spin down). The band structure, Eq. (5), is plotted in Fig. 2(a) for different values of the exchange energy $\Delta_{\text {ex }}$. One obtains the usual gapped electronic structure of antiferromagnets. (a)

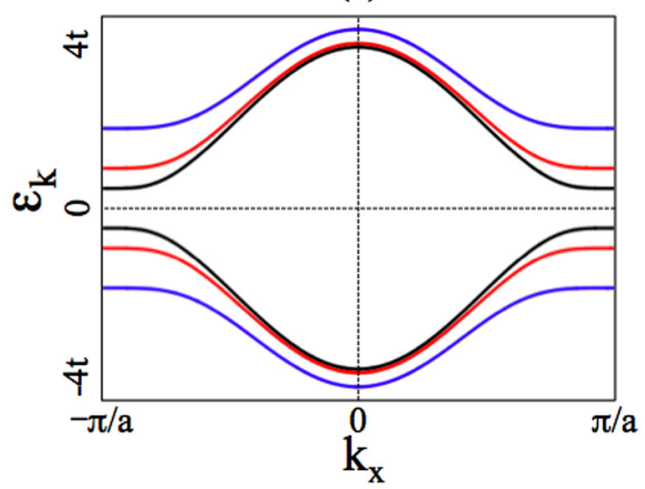

(b)

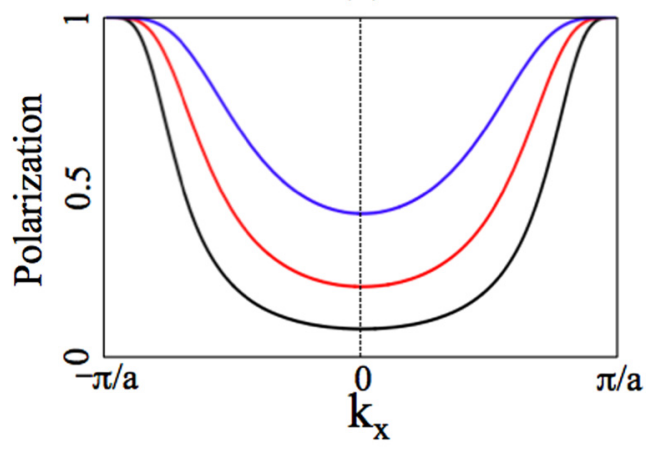

FIG. 2. (a) Band structure and (b) sublattice-resolved spin polarization of a two-dimensional G-type antiferromagnet for $\Delta_{\mathrm{ex}}=0.5 t$ (black curves), $\Delta_{\mathrm{ex}}=t$ (red curves), $\Delta_{\mathrm{ex}}=2 t$ (blue curves), and $k_{z}=0$.

Upon increasing the exchange, the band gap increases and the bandwidth is compressed.

Although spin-up and spin-down subbands are degenerate, one can define a local spin polarization on each sublattice. The density of states on sublattice $\eta(\eta=1$ corresponds to sublattice A and $\eta=-1$ corresponds to sublattice B) is indeed

$$
\mathcal{N}_{s, \sigma}^{\eta}=\frac{1}{2}\left(1+s \eta \sigma \frac{\Delta_{\mathrm{ex}}}{\epsilon}\right) \int \frac{d^{2} \mathbf{k}}{4 \pi^{2}} \delta\left(\epsilon-\epsilon_{k}^{s}\right),
$$

which produces a polarization on sublattice $\eta$ and band $s$,

$$
P_{s}^{\eta}=\left(\mathcal{N}_{s, \uparrow}^{\eta}-\mathcal{N}_{s, \downarrow}^{\eta}\right) /\left(\mathcal{N}_{s, \uparrow}^{\eta}+\mathcal{N}_{s, \downarrow}^{\eta}\right)=s \eta \Delta_{\mathrm{ex}} / \epsilon
$$

Therefore, the sublattice-resolved polarization is perfect $\left(P_{s}^{\eta}= \pm 1\right)$ at the band edges $\left(\epsilon= \pm \Delta_{\mathrm{ex}}\right)$ and decreases to a minimum at the extrema of the bands, as illustrated in Fig. 2(b). Since $\epsilon_{k}^{s}$ remains finite, the minimum polarization never vanishes. It is also remarkable that the polarization is essentially flat, i.e., energy independent, close to $k=0$. This trend is opposite to that of ferromagnets, whose density-of-states polarization reads $P=\left(\sqrt{\epsilon+\Delta_{\mathrm{ex}}}-\sqrt{\epsilon-\Delta_{\mathrm{ex}}}\right) /\left(\sqrt{\epsilon+\Delta_{\mathrm{ex}}}+\right.$ $\left.\sqrt{\epsilon-\Delta_{\mathrm{ex}}}\right)$ and decreases when the energy increases away from the bottom of the bands. These features are essential to understand the robustness of spin torque against disorder in the tunnel barrier, as discussed further below. 
(a)

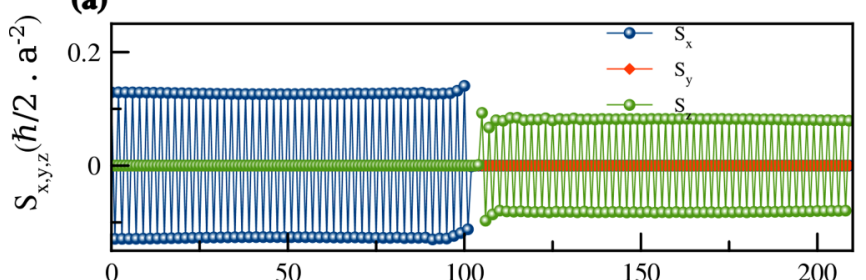

(b)

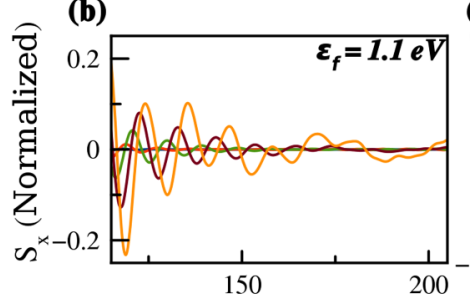

(c)

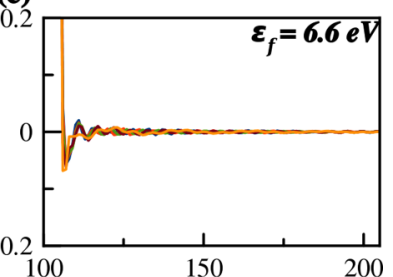

(d)

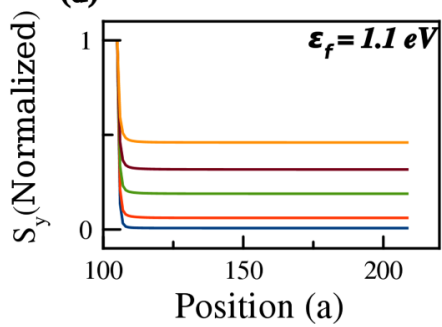

(e)

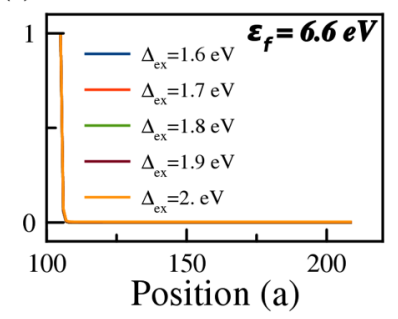

FIG. 3. (a) Spacial profile of the three components of the spin density $S_{x}$ (blue symbols), $S_{y}$ (red symbols), and $S_{z}$ (green symbols) throughout the junction. The tunnel barrier is located between position 100 and position 103. Parameters are $\epsilon_{f}=1.1 \mathrm{eV}$ and $\Delta_{\mathrm{ex}}=2 \mathrm{eV}$. Spatial profiles (b, c) of $S_{x}$ and (d, e) of $S_{y}$ in the right antiferromagnet, normalized to their magnitude at the right interface, at different exchange energies $\Delta_{\mathrm{ex}}$ and for $\epsilon_{f}=1.1 \mathrm{eV}(\mathrm{b}, \mathrm{d})$ and $\epsilon_{f}=6.6 \mathrm{eV}$ (c, e). The bias voltage is $0.6 \mathrm{eV}$ for all the calculations. Note that the solid blue line in (b) and (c) is not visible due to the very strong dephasing.

\section{B. Spin density profile}

Let us now compute the spin torque components at antiferromagnetic tunnel junctions. In the following, we fix the Néel order parameter direction of the left antiferromagnet along the $\mathbf{p}=\mathbf{x}$ direction, while that of the right antiferromagnet points along the $\mathbf{n}=\mathbf{z}$ direction, as illustrated in Fig. 1. To get better insight into the physics at stake, we consider two band filling situations: (i) $\epsilon_{f}=1.1 t$, where the Fermi energy is located in the middle of the bottom band; and (ii) $\epsilon_{f}=6.6 t$, where the Fermi energy is located in the middle of the top band (see Fig. 1). In the following, we take $t=1 \mathrm{eV}$ and the Fermi energy is defined from the bottom of the valence band.

With a finite bias voltage, electrons originating from the left antiferromagnetic electrode acquire a staggered spin density along $\mathbf{x}$ that is injected into the right electrode, as represented by the blue symbols in Fig. 3(a). In the right, downstream antiferromagnet the itinerant electron spins reorient along the local Néel order parameter, i.e., along the $\mathbf{z}$ direction [see green symbols in Fig. 3(a)]. During this reorientation, the spin density component transverse to the local Néel vector is transferred to the local magnetic moments of the right antiferromagnet. The two spin components transverse to the local Néel order parameter of the right layer are reported
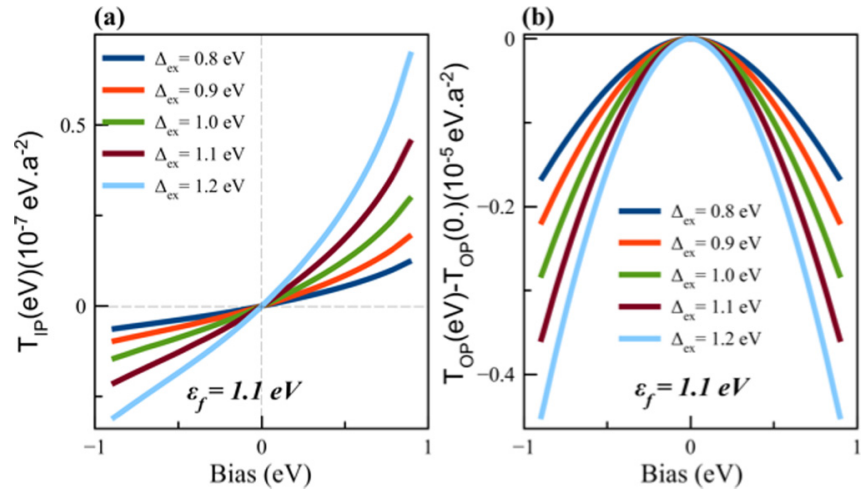

FIG. 4. Bias dependence of (a) in-plane and (b) out-of-plane torques at an AF-MTJ, calculated in the right antiferromagnet for different exchange energies $\Delta_{\mathrm{ex}}$ and $\epsilon_{f}=1.1 \mathrm{eV}$.

in Figs. 3(b) and 3(d) and Figs. 3(c) and 3(e) at different exchange energies $\Delta_{\mathrm{ex}}$ and for $\epsilon_{f}=1.1 \mathrm{eV}$ and $\epsilon_{f}=6.6 \mathrm{eV}$, respectively. For the sake of readability, we report the value of the spin densities normalized to their magnitude at the right interface (actual values of the torque are reported in Fig. 4).

The spin density $S_{x}$ displays a clear oscillatory decay [Fig. 3(b)], which resembles the behavior observed in F-MTJs (see, e.g., Ref. [10]) but is in sharp contrast with our previous calculations in metallic spin valves [24], where no such decay is observed. The decay increases when the Fermi energy is increased to $\epsilon_{f}=6.6 \mathrm{eV}$ [Fig. 3(c)] and when the exchange energy $\Delta_{\text {ex }}$ is decreased. We attribute this decay to spin dephasing arising from destructive interference between incoming electrons. Indeed, tunneling involves interference between different subbands below the Fermi level. Increasing the Fermi level from $1.1 \mathrm{eV}$ [Fig. 3(b)] to $6.6 \mathrm{eV}$ [Fig. 3(c)] increases the number of subbands involved in the tunneling process and thereby enhances the spin dephasing. Furthermore, reducing the exchange $\Delta_{\text {ex }}$ widens the bandwidth (see Fig. 2), which also participates in the enhancement of the destructive interference by allowing more states to tunnel.

The $S_{y}$ component presents a markedly different behavior [Fig. 3(d)]. It also decays away from the interface but does not present oscillations. As a matter of fact, while $S_{x}$ arises from the direct injection of the staggered spin density from the left to the right antiferromagnet, $S_{y}$ stems from the precession of $S_{x}$ about the local staggered magnetic moments. This staggered precession results in a uniform $S_{y}$ component that presents the same decaying characteristics as $S_{x}$. Moreover, in the case $\epsilon_{f}=1.1 \mathrm{eV}, S_{y}$ reaches a constant value away from the interface, while for $\epsilon_{f}=6.6 \mathrm{eV}$, it completely vanishes within a few atomic planes [Fig. 3(e)]. The latter is also a consequence of strong spin dephasing.

\section{Voltage dependence}

We now turn our attention towards the bias dependence of the spin transfer torque. From Fig. 3, one can anticipate that $S_{x}$ (which produces the out-of-plane torque, $\sim \mathbf{n} \times \mathbf{p}$ ) provides a dominant staggered spin density since it oscillates away from the interface. This staggered spin density corresponds to an exchange torque in our denomination in Ref. [24] and enables 
manipulation of the Néel order parameter [33]. In contrast, $S_{y}$ [which produces the in-plane torque, $\sim \mathbf{n} \times(\mathbf{p} \times \mathbf{n})$ ] provides a dominant uniform spin density since it has the same sign on the two opposite sublattices. This uniform spin density corresponds to a "coherent" torque in our denomination in Ref. [24] and acts like an external field on the antiferromagnetic order parameter. In other words, the two dominant torques exerted on the right antiferromagnetic electrode are

$$
\begin{gathered}
\mathbf{T}_{\mathrm{R}}^{\mathrm{u}}=T_{\mathrm{OP}} \mathbf{n} \times \mathbf{p}, \\
\mathbf{T}_{\mathrm{R}}^{\mathrm{st}}=T_{\mathrm{IP}} \mathbf{n} \times(\mathbf{p} \times \mathbf{n}),
\end{gathered}
$$

where

$$
\begin{aligned}
& T_{\mathrm{OP}}=2 \Delta_{\mathrm{ex}} \sum_{i \in \Omega_{R}}\left(S_{x}\left(x_{2 i-1}, z_{i}\right)-S_{x}\left(x_{2 i}, z_{i}\right)\right), \\
& T_{\mathrm{IP}}=-2 \Delta_{\mathrm{ex}} \sum_{i \in \Omega_{R}}\left(S_{y}\left(x_{2 i-1}, z_{i}\right)+S_{y}\left(x_{2 i}, z_{i}\right)\right) .
\end{aligned}
$$

In Eqs. (9) and (10), $\mathbf{T}_{\mathrm{R}}^{\mathrm{u}}$ is the uniform torque stemming from $S_{x}$, while $\mathbf{T}_{\mathrm{R}}^{\mathrm{st}}$ is the staggered torque stemming from $S_{y}$. In Eqs. (11) and (12), the subscript OP (IP) stands for the out-ofplane (in-plane) torque component, and $\Omega_{R}$ is the volume of the right antiferromagnetic layer. The unit is $\mathrm{eV} \cdot a^{-2}$, where $a$ is the lattice parameter (typically $0.4 \mathrm{~nm}$ ). These torques are reported in Figs. 4(a) and 4(b), respectively, for $\epsilon_{f}=1.1 \mathrm{eV}$ as a function of the bias voltage.

The bias dependence of these two torques is very similar to what is usually observed in F-MTJs [10-13]: the in-plane torque displays a bias dependence of the form $T_{\mathrm{IP}}=a_{1} V+$ $a_{2} V^{2}$, while the out-of-plane torque is mostly quadratic, $T_{\mathrm{OP}}=$ $b_{0}+b_{2} V^{2}$. The latter, which is insensitive to the bias polarity, dominates in antiferromagnetic tunnel junctions. If one introduces structural asymmetries in the junction, additional linear dependence should appear $[13,18,19]$. Remarkably, the magnitude of the torques reported in Fig. 4 is comparable to that reported for F-MTJs with similar parameters (see, e.g., Ref. [11]). Following Ref. [33], the critical current needed to switch the Néel order parameter of a uniaxial antiferromagnet reads $j_{c}^{\mathrm{AF}}=(\gamma \alpha / \xi) \sqrt{H_{k} H_{E}}$, where $\alpha$ is the phenomenological damping parameter, $\xi=\partial_{j} T$ is the torque efficiency, $H_{k}$ is the magnetic anisotropy, and $H_{E}$ is the antiferromagnetic exchange. For the sake of comparison, the critical switching current in ferromagnetic spin valves reads $j_{c}^{\mathrm{F}}=(\gamma \alpha / \xi) H_{k}$ [41]. Therefore, $j_{c}^{\mathrm{AF}}$ scales with the antiferromagnetic resonance frequency, which is typically two orders of magnitude larger than the ferromagnetic resonance. As a result, the critical switching current in antiferromagnetic junctions is expected to be two orders of magnitude larger than in ferromagnetic junctions, which is in agreement with the estimation of Cheng et al. [42] and still achievable experimentally.

Finally, following the angular momentum conservation law, the torque exerted on the left antiferromagnet simply reads

$$
\begin{gathered}
\mathbf{T}_{\mathrm{L}}^{\mathrm{u}}=-T_{\mathrm{OP}} \mathbf{n} \times \mathbf{p}, \\
\mathbf{T}_{\mathrm{L}}^{\mathrm{st}}=T_{\mathrm{IP}} \mathbf{p} \times(\mathbf{n} \times \mathbf{p}),
\end{gathered}
$$

where $\mathbf{n}$ and $\mathbf{p}$ play the role of the polarizer and free layer magnetic order directions, respectively.

\section{Effect of the disorder}

In realistic materials, a wide range of effects contributes to momentum and spin scattering: dislocations, grain boundaries, vacancies and substitutional impurities, canted magnetic moments, and interfacial roughness, but also phonons and magnons. These various imperfections have a different impact on spin transport depending on the nature of the orbitals involved in the scattering process (e.g., more localized $d$ orbitals versus more delocalized $s$ and $p$ orbitals). These effects are difficult to implement in our simple two-band square lattice system and in this work we choose to model disorder as random spatial fluctuations of the on-site energy. This on-site potential randomizes the linear momentum of the carriers and constitutes a basic test of spin transport robustness against disorder, as previously demonstrated in the case of metallic spin valves [24].

To implement disorder in our system, we follow the same procedure as in Ref. [24] and introduce a random on-site potential $\gamma_{i}$ such that $\gamma_{i} \in\left[-\frac{\Gamma}{2}, \frac{\Gamma}{2}\right]$, where $\Gamma$ is the disorder strength [43]. At this stage, the computation becomes extremely demanding, as both a large disorder configurational average and an accurate energy integration are required. In order to ensure the good convergence of our calculation, the Fermi energy is taken at $\epsilon_{f}=6.6 \mathrm{eV}$ such that a large number of modes is present in the system, thereby increasing spin dephasing and improving numerical accuracy.

\section{Disorder in metallic leads}

Let us first introduce disorder in the antiferromagnetic leads. Figures 5 and 6 display the spatial profile of $S_{x}$ and $S_{y}$, respectively, for different disorder strengths and exchange energies. The clean regime is also reported for comparison (solid lines in Figs. 5 and 6). Symbols represent the disordered regime, with $\Gamma$ ranging from 0.1 to $0.4 \mathrm{eV}$. Since the spin density decreases dramatically within two monolayers from the interface (see Fig. 3), we focus on the impact of disorder
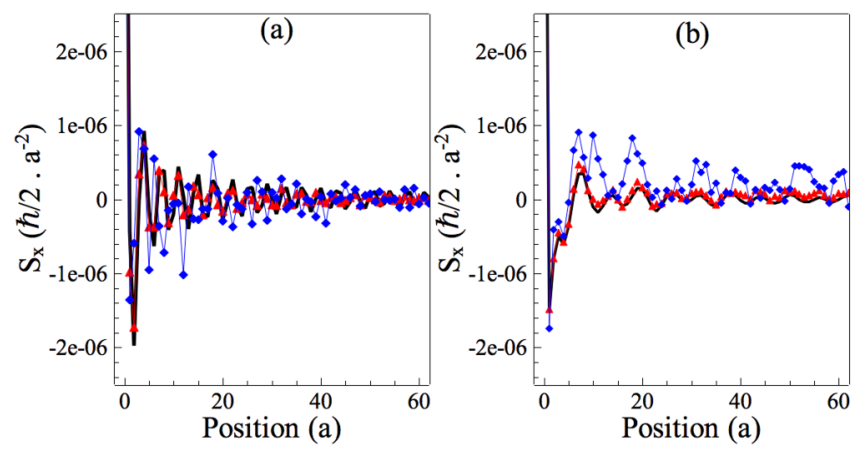

FIG. 5. (a) Spatial profile of $S_{x}$ in the right antiferromagnet for $\Gamma=0$ (solid line), $0.2 \mathrm{eV}$ (red symbols), and $0.4 \mathrm{eV}$ (blue symbols) with $\Delta_{\mathrm{ex}}=1 \mathrm{eV}$. (b) Spatial profile of $S_{x}$ in the right antiferromagnet for $\Gamma=0$ (solid line), $0.1 \mathrm{eV}$ (red symbols), and $0.3 \mathrm{eV}$ (blue symbols) with $\Delta_{\mathrm{ex}}=2 \mathrm{eV}$. Calculated quantities are averaged over 2000 disorder configurations. 

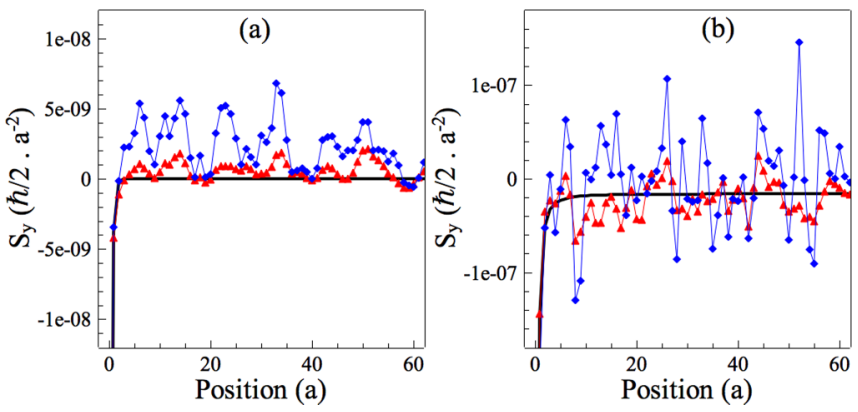

FIG. 6. (a) Spatial profile of $S_{y}$ in the right antiferromagnet for $\Gamma=0$ (solid line), $0.2 \mathrm{eV}$ (red symbols), and $0.4 \mathrm{eV}$ (blue symbols) with $\Delta_{\mathrm{ex}}=1 \mathrm{eV}$. (b) Spatial profile of $S_{y}$ in the right antiferromagnet for $\Gamma=0$ (solid line), $0.1 \mathrm{eV}$ (red symbols), and $0.3 \mathrm{eV}$ (blue symbols) with $\Delta_{\mathrm{ex}}=2 \mathrm{eV}$. Calculated quantities are averaged over 2000 disorder configurations.

on the oscillatory decay of the spin density in the bulk of the antiferromagnet. For weak disorder (red symbols) in Figs. 5(a) and 5(b), the oscillation of $S_{x}$ remains weakly affected, while increasing the disorder results in enhanced deviations (blue symbols). However, Figs. 5(a) and 5(b) show that disorder mostly affects the spin density in the bulk of the antiferromagnet, away from the interface. As a result, since the torque mostly occurs at the interface where the spatial decay is stronger, the overall torque magnitude remains only weakly affected by disorder. A similar conclusion can be drawn for $S_{y}$, displayed in Fig. 6. Again, the magnitude of $S_{y}$ is mostly affected by bulk disorder, while its value close to the interface remains robust. In conclusion, the overall impact of disorder on spin torque is much less dramatic than in metallic spin valves since in AF-MTJs the torque is mainly an interfacial effect.

\section{Disorder in the tunnel barrier}

The impact of disorder in the tunnel barrier on spin transport has been reported in the case of tunneling magnetoresistance in F-MTJs [44]. In these structures, the disorder inside the tunnel spacer is detrimental to spin transport properties since a local reduction in the barrier height or thickness enhances the tunneling current while reducing its spin polarization. In other words, the presence of disorder in the tunnel barrier introduces hot spots of weakly polarized current that dominate the magnetoresistance signal. Let us now consider the impact of disorder in the barrier on spin torque in F-MTJs. Figure 7(a) shows the torkance-or torque efficiency-defined as the torque normalized to the conductance, exerted on the right ferromagnetic layer. The torkance (proportional to the interfacial polarization) dramatically decreases in the presence of disorder, which is consistent with the behavior previously observed for tunneling magnetoresistance [44].

We now turn our attention to the effect of disorder on the spin transfer torque in AF-MTJs, reported in Fig. 7(b)

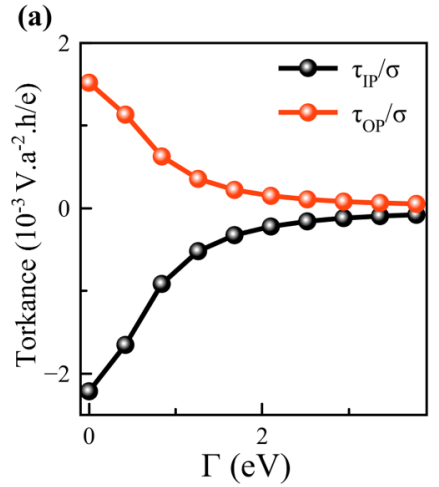

(b)

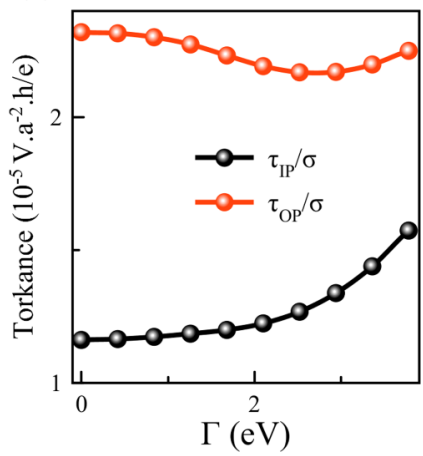

FIG. 7. Impact of disorder on the torkance components in (a) F-MTJs and (b) AF-MTJs. Black and red symbols refers to in-plane and out-of-plane components, respectively. The exchange splitting is set to $\Delta_{\mathrm{ex}}=2 \mathrm{eV}$, the width of the junctions is 10 monolayers, the bias voltage is $0.6 \mathrm{eV}$, and the torque computed here is the total torque.

[the parameters are the same as in Fig. 7(a)]. Surprisingly, the torkance remains mostly unaffected by the disorder, in sharp contrast with F-MTJs. This illustrates the major difference between AF-MTJs and F-MTJs: since up and down spins are degenerate [see Fig. 2 and related discussion], the hot spots introduced by the disorder results only in an enhancement of the tunneling current, without altering the sublattice polarization. As a consequence, the spin torque efficiency in AF-MTJs is much more robust against disorder than in F-MTJs.

\section{CONCLUSION}

In the present work we have studied the spin transfer torque in AF-MTJs using a real-space tight-binding model. We have shown that, similarly to the case of F-MTJs, the antiferromagnetic torque is interfacial and possesses both in-plane and out-of-plane torques, the former being mostly linear in bias voltage while the latter is quadratic for a symmetric system. However, two main differences have been identified. First, since only staggered spin densities are efficient in manipulating the Néel order parameter, the efficient torque is out of plane. Second, because up- and down-spin subbands are degenerate in antiferromagnets, the torque efficiency in AF-MTJs is much more robust against disorder than in F-MTJs. This shows that AF-MTJs, such as $\mathrm{Cr} / \mathrm{MgO} / \mathrm{Cr}[39,40]$, are solid candidates for the realization of spin transfer torque in antiferromagnets.

\section{ACKNOWLEDGMENT}

A.M. acknowledges the financial support of the King Abdullah University of Science and Technology (KAUST) through the Office of Sponsored Research (OSR; Grant No. OSR-2015-CRG4-2626).
[1] J. C. Slonczewski, J. Magn. Magn. Mater. 159, L1 (1996).

[2] L. Berger, Phys. Rev. B 54, 9353 (1996).
[3] D. C. Ralph and M. D. Stiles, J. Magn. Magn. Mater. 320, 1190 (2008). 
[4] J. Z. Sun, J. Magn. Magn. Mater. 202, 157 (1999).

[5] J. A. Katine, F. J. Albert, R. A. Buhrman, E. B. Myers, and D. C. Ralph, Phys. Rev. Lett. 84, 3149 (2000).

[6] D. Chiba, Y. Sato, T. Kita, F. Matsukura, and H. Ohno, Phys. Rev. Lett. 93, 216602 (2004); Y. Huai, F. Albert, P. Nguyen, M. Pakala, and T. Valet, Appl. Phys. Lett. 84, 3118 (2004); G. D. Fuchs et al., ibid. 85, 1205 (2004).

[7] S. I. Kiselev, J. C. Sankey, I. N. Krivorotov, N. C. Emley, R. J. Schoelkopf, R. A. Buhrman, and D. C. Ralph, Nature 425, 380 (2003).

[8] W. H. Rippard, M. R. Pufall, S. Kaka, S. E. Russek, and T. J. Silva, Phys. Rev. Lett. 92, 027201 (2004).

[9] M. D. Stiles and A. Zangwill, Phys. Rev. B 66, 014407 (2002).

[10] A. Manchon, N. Ryzhanova, N. Strelkov, A. Vedyayev, M. Chshiev, and B. Dieny, J. Phys.: Condens. Matter 20, 145208 (2008); 19, 165212 (2007).

[11] I. Theodonis, N. Kioussis, A. Kalitsov, M. Chshiev, and W. H. Butler, Phys. Rev. Lett. 97, 237205 (2006); A. Kalitsov, M. Chshiev, I. Theodonis, N. Kioussis, and W. H. Butler, Phys. Rev. B 79, 174416 (2009).

[12] J. Xiao, G. E. W. Bauer, and A. Brataas, Phys. Rev. B 77, 224419 (2008).

[13] M. Wilczynski, J. Barnas, and R. Swirkowicz, Phys. Rev. B 77, 054434 (2008).

[14] C. Heiliger and M. D. Stiles, Phys. Rev. Lett. 100, 186805 (2008).

[15] J. C. Sankey, Y.-T. Cui, J. Z. Sun, J. C. Slonczewski, R. A. Buhrman, and D. C. Ralph, Nat. Phys. 4, 67 (2008).

[16] H. Kubota, A. Fukushima, K. Yakushiji, T. Nagahama, S. Yuasa, K. Ando, H. Maehara, Y. Nagamine, K. Tsunekawa, D. D. Djayaprawira, N. Watanabe, and Y. Suzuki, Nat. Phys. 4, 37 (2008).

[17] S.-C. Oh, S.-Y. Park, A. Manchon, M. Chshiev, J.-H. Han, H.-W. Lee, J.-E. Lee, K.-T. Nam, Y. Jo, Y.-C. Kong, B. Dieny, and K.-J. Lee, Nat. Phys. 5, 898 (2009).

[18] A. Manchon, S. Zhang, and K.-J. Lee, Phys. Rev. B 82, 174420 (2010).

[19] Y.-H. Tang, N. Kioussis, A. Kalitsov, W. H. Butler, and R. Car, Phys. Rev. Lett. 103, 057206 (2009); Phys. Rev. B 81, 054437 (2010).

[20] A. Manchon and S. Zhang, Phys. Rev. B 79, 174401 (2009).

[21] A. S. Nunez, R. A. Duine, P. Haney, and A. H. MacDonald, Phys. Rev. B 73, 214426 (2006).

[22] Z. Wei, A. Sharma, A. S. Nunez, P. M. Haney, R. A. Duine, J. Bass, A. H. MacDonald, and M. Tsoi, Phys. Rev. Lett. 98, 116603 (2007).

[23] S. Urazhdin and N. Anthony, Phys. Rev. Lett. 99, 046602 (2007).

[24] H. B. M. Saidaoui, A. Manchon, and X. Waintal, Phys. Rev. B 89, 174430 (2014).
[25] R. A. Duine, P. M. Haney, A. S. Núñez, and A. H. MacDonald, Phys. Rev. B 75, 014433 (2007).

[26] K. M. D. Hals, Y. Tserkovnyak, and A. Brataas, Phys. Rev. Lett. 106, 107206 (2011); E. G. Tveten, A. Qaiumzadeh, and A. Brataas, ibid. 112, 147204 (2014).

[27] A. C. Swaving and R. A. Duine, Phys. Rev. B 83, 054428 (2011); J. Phys.: Condens. Matter 24, 024223 (2012).

[28] J. Zelezny, H. Gao, K. Vyborny, J. Zemen, J. Masek, A. Manchon, J. Wunderlich, J. Sinova, and T. Jungwirth, Phys. Rev. Lett. 113, 157201 (2014); Wadley et al., Science 351, 587 (2016).

[29] P. Merodio, A. Kalitsov, H. Bea, V. Baltz, and M. Chshiev, Appl. Phys. Lett. 105, 122403 (2014).

[30] B. G. Park, J. Wunderlich, X. Marti, V. Holy, Y. Kurosaki, M. Yamada, H. Yamamoto, A. Nishide, J. Hayakawa, H. Takahashi, A. B. Shick, and T. Jungwirth, Nat. Mater. 10, 347 (2011); X. Martí, B. G. Park, J. Wunderlich, H. Reichlová, Y. Kurosaki, M. Yamada, H. Yamamoto, A. Nishide, J. Hayakawa, H. Takahashi, and T. Jungwirth, Phys. Rev. Lett. 108, 017201 (2012).

[31] Y. Y. Wang, C. Song, B. Cui, G. Y. Wang, F. Zeng, and F. Pan, SPIN 3, 1350005 (2013).

[32] T. Jungwirth, X. Martí, P. Wadley, and J. Wunderlich, Nat. Nanotechnol. 11, 231 (2016).

[33] E. V. Gomonay and V. M. Loktev, Low Temp. Phys. 40, 17 (2014); Phys. Rev. B 81, 144427 (2010).

[34] C. W. Groth, M. Wimmer, A. R. Akhmerov, and X. Waintal, New J. Phys. 16, 063065 (2014).

[35] M. Wimmer, Ph.D. thesis, University of Regensburg 5 (2009).

[36] K. Prakhya, A. Popescu, and P. M. Haney, Phys. Rev. B 89, 054421 (2014).

[37] C. Tiusan, J. Faure-Vincent, C. Bellouard, M. Hehn, E. Jouguelet, and A. Schuhl, Phys. Rev. Lett. 93, 106602 (2004).

[38] P.-J. Zermatten, G. Gaudin, G. Maris, M. Miron, A. Schuhl, C. Tiusan, F. Greullet, and M. Hehn, Phys. Rev. B 78, 033301 (2008).

[39] M.-A. Leroy, A. M. Bataille, F. Bertran, P. Le Fèvre, A. TalebIbrahimi, and S. Andrieu, Phys. Rev. B 88, 205134 (2013).

[40] M.-A. Leroy, A. M. Bataille, B. Dkhil, F. Porcher, A. Barbier, Vincent L. R. Jacques, Y. Lu, C. Bellouard, T. Hauet, S. Ravy, J. Herrero-Martin, C. Gatel, K. Bouzehouane, A. Gukasov, S. Andrieu, and C. Tiusan, Phys. Rev. B 90, 035432 (2014).

[41] J. Grollier, V. Cros, H. Jaffres, A. Hamzic, J. M. George, G. Faini, J. B. Youssef, H. LeGall, and A. Fert, Phys. Rev. B 67, 174402 (2003).

[42] R. Cheng, D. Xiao, and A. Brataas, Phys. Rev. Lett. 116, 207603 (2016).

[43] S. Borlenghi, V. Rychkov, C. Petitjean, and X. Waintal, Phys. Rev. B 84, 035412 (2011).

[44] E. Y. Tsymbal, O. N. Mryasov, and P. R. LeClair, J. Phys.: Condens. Matter 15, R109 (2003). 